\title{
High-Performance CMOS Current Mirrors: Application to Linear Voltage-to-Current Converter Used for Two-Stage Operational Amplifier
}

\author{
Radwene Laajimi, Mohamed Masmoudi \\ Department of Electrical Engineering Electronics, Micro-Technology and Communication (EMC) Research Group Sfax (ENIS), \\ University of Sfax, Sfax, Tunisia \\ Email: radwene_fac@hotmail.fr
}

Received May 3, 2012; revised June 28, 2012; accepted July 5, 2012

\begin{abstract}
This paper presents two schemes of high performance CMOS current mirror, one of them is used for operational transconductance amplifier (OTA) in analog VLSI systems. The linearity, output impedance, bandwidth and accuracy are the most parameters to determine the performance of the current mirror. Here a comparison of two architectures based on same architecture of the amplifier is presented. This comparison includes: linearity, output impedance, bandwidth and accuracy. These two circuits are validated with simulation in technology AMS $0.35 \mu \mathrm{m}$. An operational amplifier based on the adapted current mirror is proposed. Its frequency analysis with large bandwidth is validated with the same technology.
\end{abstract}

Keywords: Analog Circuits; Current Source; Current Mirror; Low Voltage; Operational Transconductance Amplifier

\section{Introduction}

In the last few years, the demand for analog circuits which can operate at low voltage is an established fact and does not need any further justification. In particular structures of current mirrors which have increased rapidly and become one of the most interesting areas of research [1]. Many configurations of current mirror are discussed and used for many applications. Especially cascode current mirror which is one of the main building blocks of analog and mixed-signal integrated circuits. For low voltage design circuit and high speed application, the important parameters to determine high performance current mirror are [2-4]:

- Low input and output voltage.

- Low input impedance.

- High output impedance.

- Minimum error of copying accuracy and settling time. The four transistors circuit shown in Figure 1(a) is a simple cascode current mirror and it is characterized by moderately low input and output voltages.

Equation (1) of input voltage is:

$$
V_{\text {in }}=2 V_{\text {DSAT }}+2 V_{T}
$$

Equation (2) of output voltage is:

$$
V_{\text {out }}=2 V_{\text {DSAT }}+V_{T}
$$

$\left(V_{\text {DSAT }}\right.$ and $V_{T}$ denote is the minimum drain-source saturation voltage and the transistor's threshold voltage.)
This circuit has higher output impedance than the simple current mirror and moderately high input impedance.

Equation (3) of input impedance is:

$$
Z_{\text {in }}=\frac{1}{g m_{11}}+\frac{1}{g m_{12}}
$$

Equation (4) of output impedance is:

$$
Z_{\text {out }}=\frac{g m_{9}}{g o_{9} \cdot g o_{10}}
$$

(where $g m$ and go are the small-signal transconductance gain and the conductance of the MOS transistor, respectively.)

A simple variation of this circuit is shown in Figure 1(b) [5]. In this scheme the variable voltage $V_{a r}$ with resistance R1 are added. They are injected between the drain of transistor M12 and the source of transistor M11. The drain source voltage of the mirror transistor M12 is forced to a constant value by means of transistor M11, current $I_{\text {in }}$ and variable voltage $V_{a r}$. Thus, on one hand, a reduction of the input impedance by the gain of M11. On the other hand, it reduces the input voltage requirements. In this case, the input voltage can be set to its minimum value by proper selection of the variable voltage Var. Hence, this scheme achieves low input impedance with low input voltage requirements but with some limitation due to the difference between the current in M11 and M9. This difference causes a mismatch in the drain source 


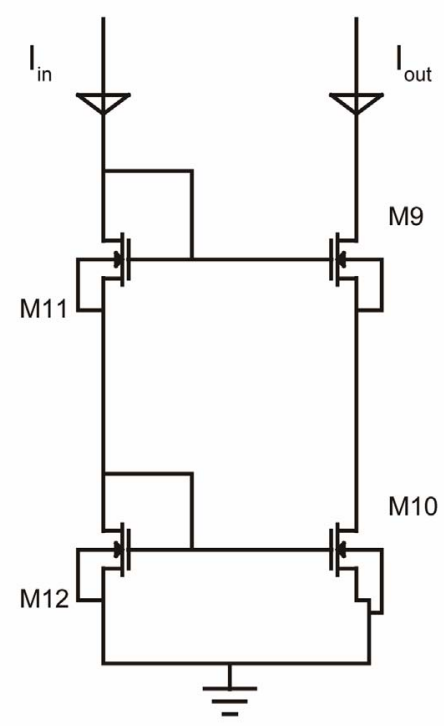

(a)

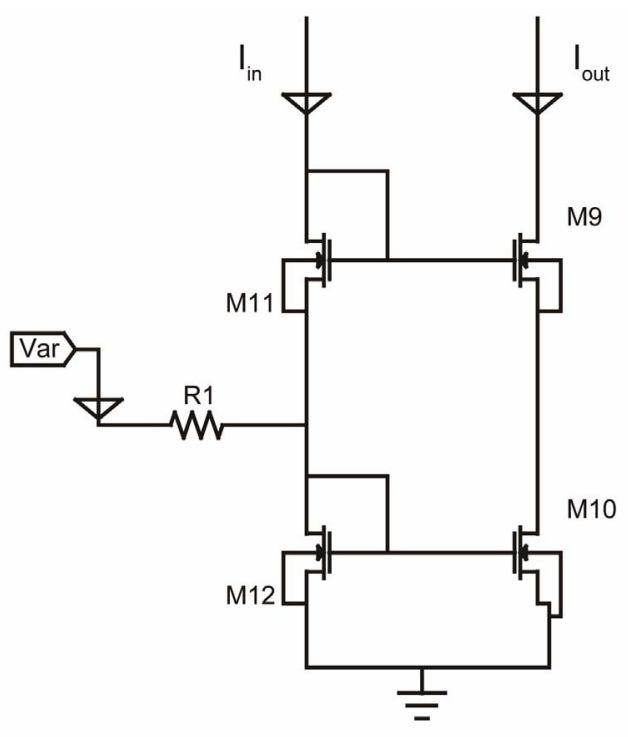

(b)

Figure 1. Current mirror configuration. (a) Simple cascode current mirror; (b) Cascode current mirror with regulated voltage.

voltage of M10 and M12 and results in a loss of accuracy, so degraded linearity (due to channel length modulation).

To overcome this limitation, we are proposing two novels structures of current mirror which offers both the low compliance voltage and a minimum error of copying accuracy. The two proposed approaches are based on using a differential amplifier to compare the drain voltages of the input and output mirror transistors, to force the equality between these voltages, thereby improving the accuracy of the current copy, and improving linearity.

The paper is organized as follows. The second section presents the amplifiers structures used for voltage to cur- rent (V-I) converter. In the third section, the design of proposed version 1 of $\mathrm{V}$-I converter is presented with simulations results. The proposed version 2 of $\mathrm{V}$-I converter is presented in section four. Section 5 presents an application of V-I converter in two-stage operational amplifier. Conclusion is drawn in the last section.

\section{Amplifier Structures}

In order to achieve high current copy accuracy, it is necessary to use an amplifier between the mirror's input and output transistors.

The amplifier architectures are: simple differential amplifier [6] and amplifier proposed by [5]. As shown in Figure 2(a), this structure is formed by the input differential pair (MP1 and MP2) and the active charge (MN1 and MN2). The Figure 2(b) shows a differential amplifier with two MOS transistors (MN1 and MN2) in which $\mathrm{MN} 1$ is operated in the weak version region (source gate voltage of MN1 equal to zero). This version can operate either in the linear region or in the saturation region for achieving low voltage and low consumption.

Moreover, the amplifier, which has many transistors, causes the increase of the power dissipation and the chip area. The advantages of the scheme proposed by [7] are low voltage operation, small chip area, high output resistance and no bias current. For this reason we use this structure in our two versions of voltage to current converter which are more described in the following paragraph.

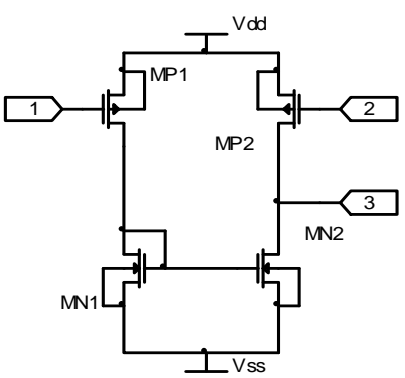

(a)

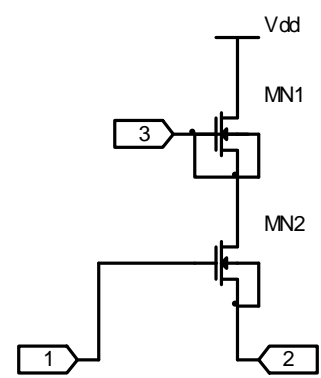

(b)

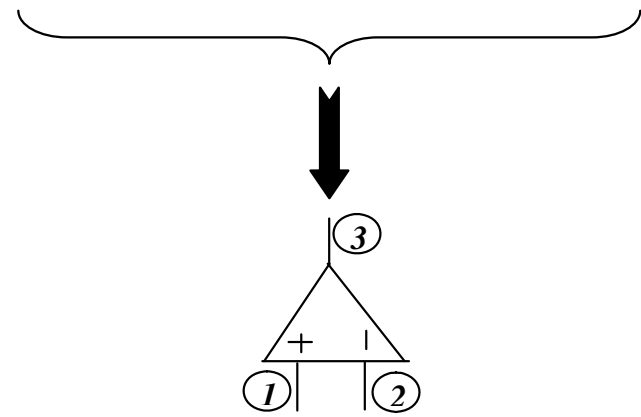

Figure 2. Amplifier configuration. (a) Simple differential amplifier structure [6]; (b) Two-transistor apmlifier structure [7]. 


\section{Proposed Version 1 of Voltage to Current Converter}

\subsection{Description}

According to Figure 3 the version 1 of V-I converter is composed of three blocks: polarisation, correction and output load.

The polarisation block is formed of four transistors (MP1, MP2, MP3, MP4) with variable input voltage $V_{\text {in }}$ and input resistance $R_{\text {in }}$. The correction block is composed of three transistors (M1, M2, MC2). It characterized by minimum error of copying accuracy due to the equality of drain source voltage between M1 and M2. This equality thanks to an amplifier between the mirror's input and output transistors M1 and M2 and causes high accuracy and good linearity. High output impedance is obtained by the output current of amplifier and passes to the gate of transistor MC2. The output load block is a simply resistance $\left(R_{\text {out }}\right)$.

This scheme offers a comparison between drain source voltage of M1 and M2 by using a differential amplifier to provide higher accuracy of the current copy. The input injection current signal $I_{\text {in }}$ is replaced by another source formed by variable input voltage $V_{\text {in }}$ with resistance $R_{\text {in }}$. On the one hand, we obtain a reduction of the input impedance which given by this relation:

Equation (5) of input impedance is:

$$
Z_{\text {in }}=\frac{1}{g m_{1} \cdot A_{\text {olinp }}}
$$

Equation (6) presents moderate output impedance

$$
Z_{\text {out }}=r O_{2} \cdot A_{\text {olout }}=r O_{2} \cdot A v_{\mathrm{MC} 2} \cdot A v_{\text {omp }}
$$

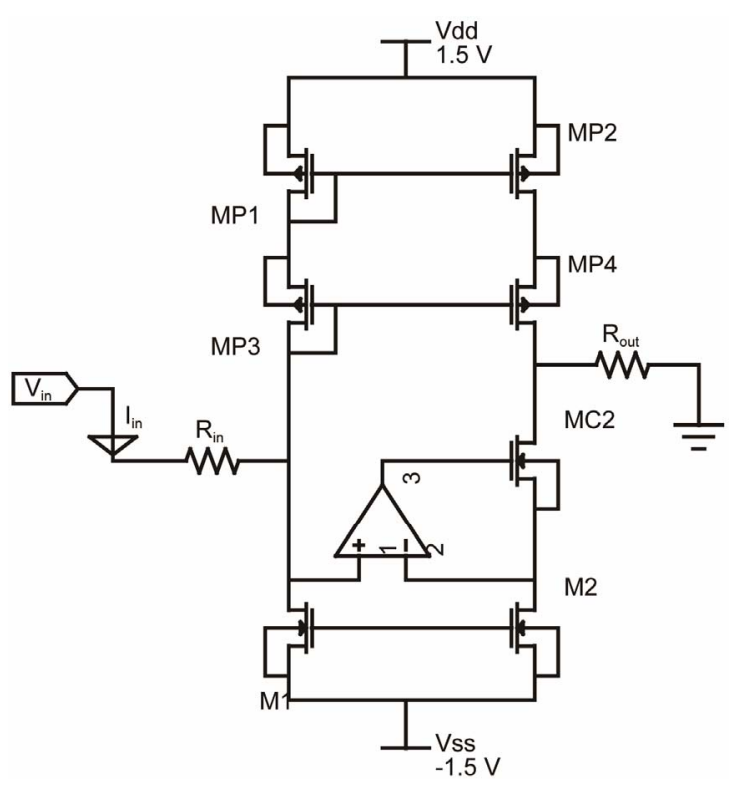

Figure 3. Proposed version 1 of voltage to current converter. where $\mathrm{gm}$ and ro are the small-signal transconductance gain and the output resistance of the MOS transistors. In this case we assume that the amplifier have an input open-loop gain $A_{\text {olinp }}$ and output open-loop gain $A_{\text {olout }}$ $\left(A_{\text {olout }}=A v_{\mathrm{MC} 2} \cdot A v_{\text {omp }}\right) . A v_{\mathrm{MC} 2}$ and $A v_{\text {omp }}$ denote the voltage gain of the transistor $\mathrm{MC} 2$ and amplifier gain respectively.

On the other hand, the drain source voltage of the mirror transistor M1 achieves a small constant value thanks to current source $I_{\text {in }}$ and variable voltage source $V_{\text {in }}$. Drain source voltage can be decreasing to a minimum value, by selection of $V_{\text {in }}$ and consequently a very low input voltage of the circuit.

\subsection{Simulations Results}

Different schemes are simulated using Tspice based on BSIM3V3 transistor model for the technology AMS 0.35 $\mu \mathrm{m}$ at $\pm 1.5 \mathrm{~V}$ power supply voltage.

Tspice simulations are carried for an input voltage $V_{\text {in }}$ varied from $-1.1 \mathrm{~V}$ to $0 \mathrm{~V}$, Figure 4 shows the DC characteristic for the $\mathrm{V}$-I converter for different values of resistance $\left(R_{\text {out }}=100 \Omega, R_{\text {out }}=1 \mathrm{~K} \Omega, R_{\text {out }}=5 \mathrm{~K} \Omega\right)$ in which the full input voltage swing capability is evident with truly linearity.

As shown in Figure 5, from DC output characteristics simulations, the output resistance presents a moderate value of $0.18 \mathrm{M} \Omega$.

The Figure 6 shows the AC characteristic of the proposed V-I converter. For a resistance of $100 \Omega$, we achieved a common gain bandwidth (GBW) equal to $750 \mathrm{MHz}$ for different values of resistance $\left(R_{\text {out }}=100\right.$ $\left.\Omega, R_{\text {out }}=1 \mathrm{~K} \Omega, R_{\text {out }}=5 \mathrm{~K} \Omega\right)$ and variable gain $(A v)$ from $53 \mathrm{~dB}$ to $80.2 \mathrm{~dB}$ at minimum resistance $\left(R_{\text {out }}=100 \Omega\right)$.

According to Figure 7, the deviation of the DC output current from the ideal characteristic for different values of resistance $R_{\text {out }}$. The large error is reached for the lowest input voltage $V_{\text {in }}$ of $-1.1 \mathrm{~V}$. On the other hand the variation of $V_{\text {in }}$ between $-0.9 \mathrm{~V}$ to $0 \mathrm{~V}$ provide a small current error under $0.1 \%$ for different values of resistance $\left(R_{\text {out }}=100 \Omega, R_{\text {out }}=1 \mathrm{~K} \Omega, R_{\text {out }}=5 \mathrm{~K} \Omega\right)$. Moreover for the maximum current error of $0.5 \%, V_{\text {in }}$ varied from -1.1 $\mathrm{V}$ to $-0.95 \mathrm{~V}$ in particular for output resistance $R_{\text {out }}=5$ $\mathrm{K} \Omega$.

\section{Proposed Version 2 of Voltage to Current Converter}

\subsection{Description}

The version 2 of V-I converter is presented in Figure 8. It is like the structure of version 1 shown in Figure 3 and composed of three blocks: polarisation, correction and output load. The main difference is in the correction block. In which there are three transistors (M1, M2, MC2) 


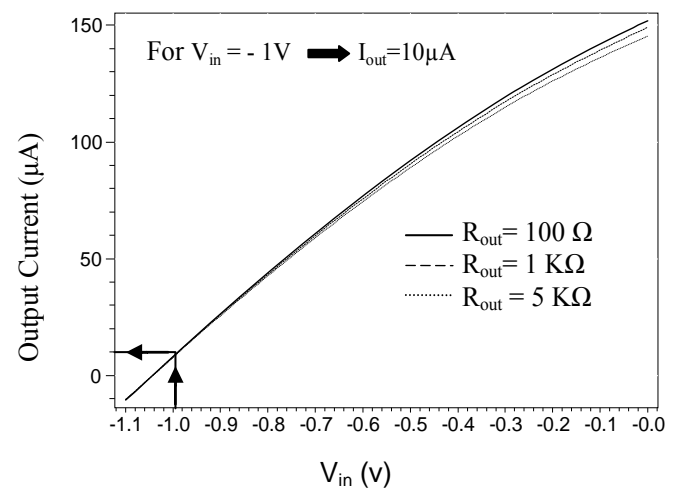

Figure 4. DC characteristics of V-I converter for different values of resistance $R_{\text {out }}$.

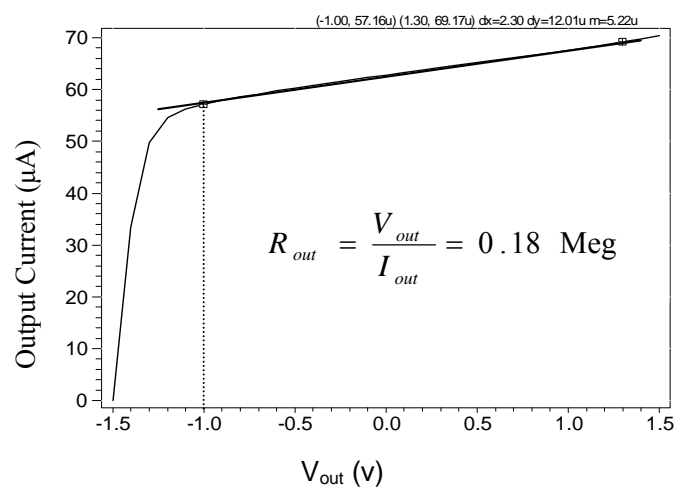

Figure 5. Output current vs output voltage variation of proposed V-I converter.

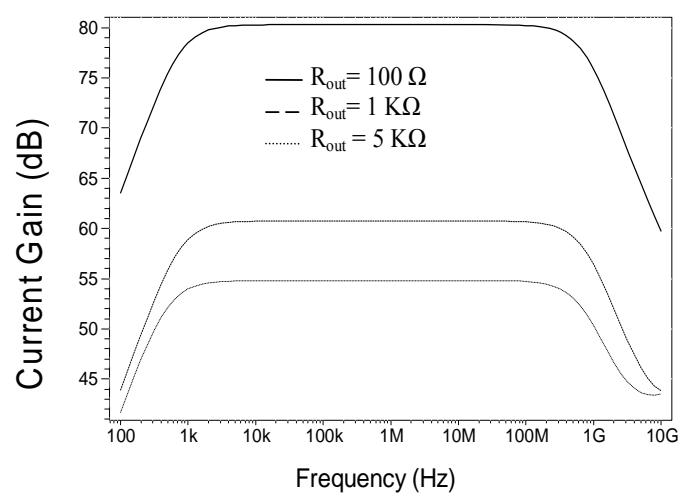

Figure 6. Frequency response of proposed V-I converter.

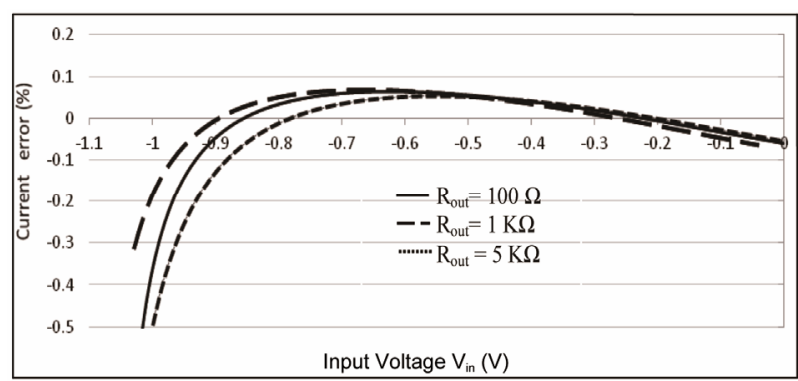

Figure 7. Current error of V-I converter.

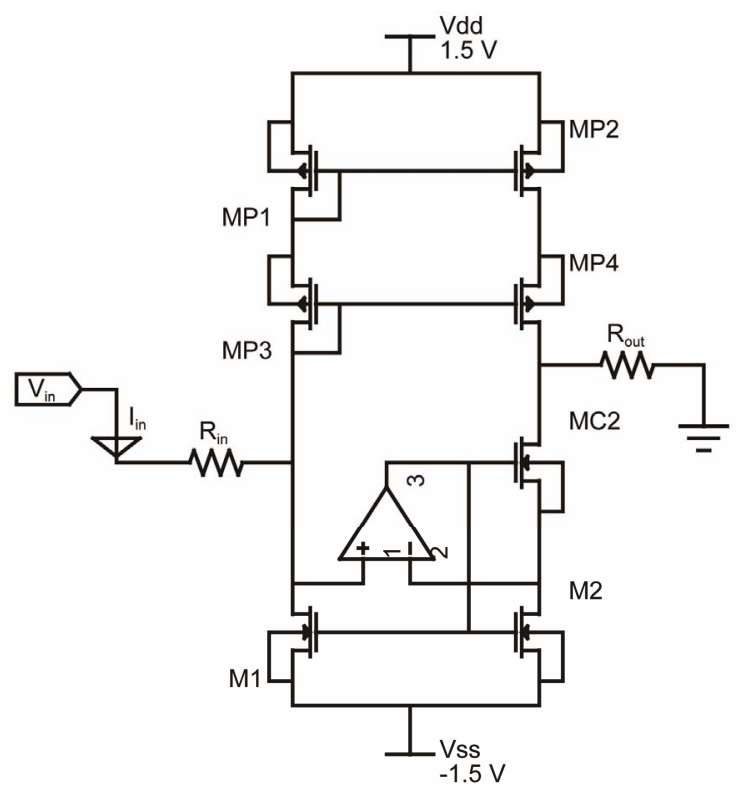

Figure 8. Proposed version 2 of voltage to current converter.

with an amplifier between M1 and M2 in order to minimise error of copying accuracy.

In this case of structure an approach is used to increase the output impedance without sacrificing the equality between input and output of drain source voltage (M1 and M2), consists in integrating a connection between the gate of transistor MC2 and the gate of transistor M2. We achieved very high output impedance due to the output current of amplifier. This current is passed through each gate of transistors MC2 and M2. Equation (8) shows the value of output impedance which is:

$$
Z_{\text {out }}=r \mathrm{O}_{2} \cdot A_{\text {olout }}=r \mathrm{O}_{2} \cdot A v_{\mathrm{MC} 2} \cdot A v_{\mathrm{M} 2} \cdot A v_{\text {omp }}
$$

$\mathrm{rO}_{2}$ denote the output resistance of the MOS transistor M2. The amplifier has an output open-loop gain $A_{\text {olout }}$. Equation (9) give this relation:

$$
A_{\text {olout }}=A v_{\mathrm{MC} 2} \cdot A v_{\mathrm{M} 2} \cdot A v_{\text {omp }}
$$

We assume that $A v_{\mathrm{MC} 2}, A v_{\mathrm{M} 2}$ and $A v_{\text {omp }}$ denote the voltage gain of the transistor $\mathrm{MC} 2, \mathrm{M} 2$ and amplifier gain respectively.

\subsection{Simulations Results}

From Tspice simulations, an input voltage $V_{\text {in }}$ varied from $-1 \mathrm{~V}$ to $0 \mathrm{~V}$, the DC characteristic for the V-I converter for different values of resistance $\left(R_{\text {out }}=100 \Omega, R_{\text {out }}\right.$ $=1 \mathrm{~K} \Omega, R_{\text {out }}=5 \mathrm{~K} \Omega$ ) is shown in Figure 9. We find that the linearity is evident for each value of resistance.

To confirm the high output resistance given by the proposed version 2 of V-I converter, Figure 10 shows the DC output characteristics simulations. The value of the output resistance in this case is equal to $0.34 \mathrm{M} \Omega$. 


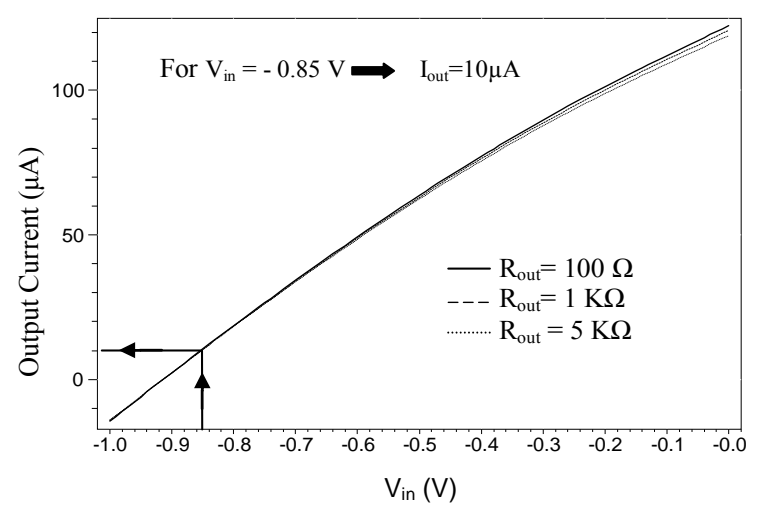

Figure 9. DC characteristics of V-I converter for different values of resistance $R_{\text {out }}$.

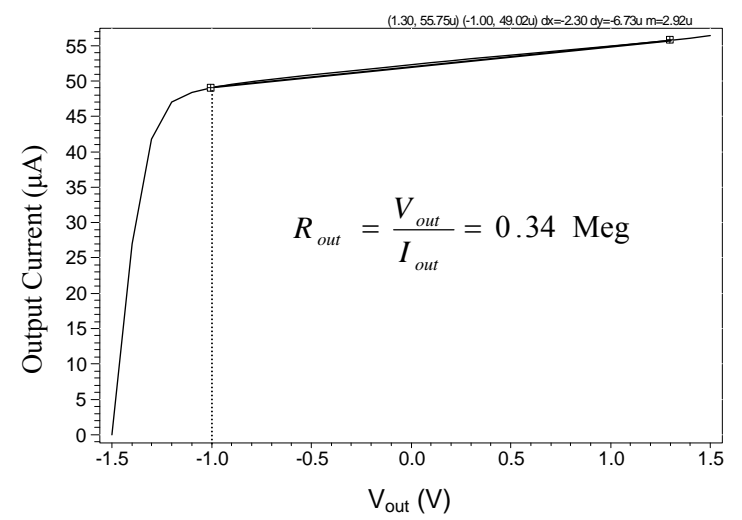

Figure 10. Output current vs output voltage variation of proposed V-I converter.

From AC characteristic, we note an improvement of the gain $(A v)$. Its maximum value is $73 \mathrm{~dB}$ but it is 80.2 $\mathrm{dB}$ for the proposed version 1 of $\mathrm{V}-\mathrm{I}$ converter. The bandwidth responses for different values of resistance are presented in Figure 11.

From Figure 12, we present a current error of V-I converter for different values of resistance $R_{\text {out }}$. On the one hand, the variation of $V_{\text {in }}$ from $-0.9 \mathrm{~V}$ to $0 \mathrm{~V}$ give the same characteristic of error for different values of resistance $\left(R_{\text {out }}=100 \Omega, R_{\text {out }}=1 \mathrm{~K} \Omega, R_{\text {out }}=5 \mathrm{~K} \Omega\right)$. On the other hand for the maximum current error of $0.35 \%, V_{\text {in }}$ is lower than $-0.95 \mathrm{~V}$.

\section{Application of V-I Converter in Two-Stage Operational Amplifier}

Voltage to current V-I converter becomes the most interesting element of interface measurement in the field of mixed signal systems [8].

The most important parameters to determine high performance of current V-I converters are:

- High linear range.

- Large bandwidth and gain.

Because the large bandwidth and gain of the proposed version 1 of V-I converter, it is possible to use this approach for Operational amplifier $[9,10]$. The Figure 13 shows a practical implementation of the two-stage Operational amplifier.

The simulated output frequency response of our application is shown in Figure 14. The bode diagram gives an open loop gain of $60 \mathrm{~dB}$ with a large $\mathrm{GBW}$ of $82 \mathrm{MHz}$, a $97 \mathrm{KHz}$ of cut-off frequency and a phase margin of $62^{\circ}$. We note that the input current passes through M8 using for polarisation is equal to $10 \mu \mathrm{A}$ and this corresponds to an input voltage $V_{\text {in }}$ of $-1 \mathrm{~V}$.

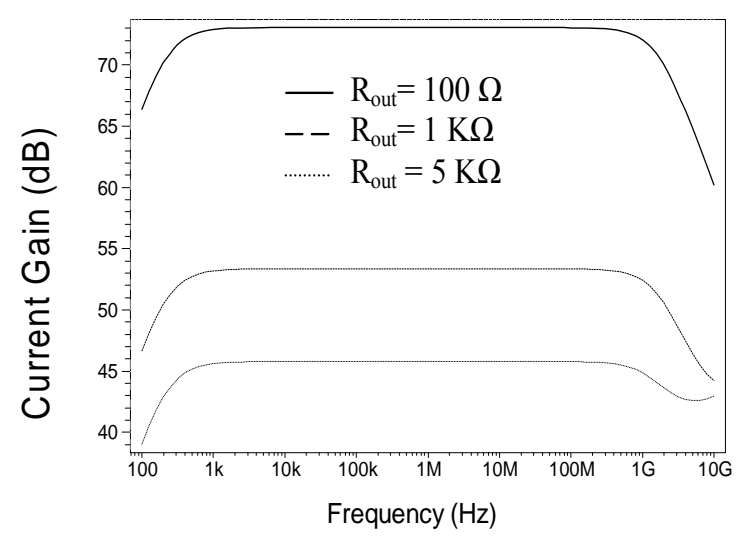

Figure 11. Frequency response of proposed V-I converter

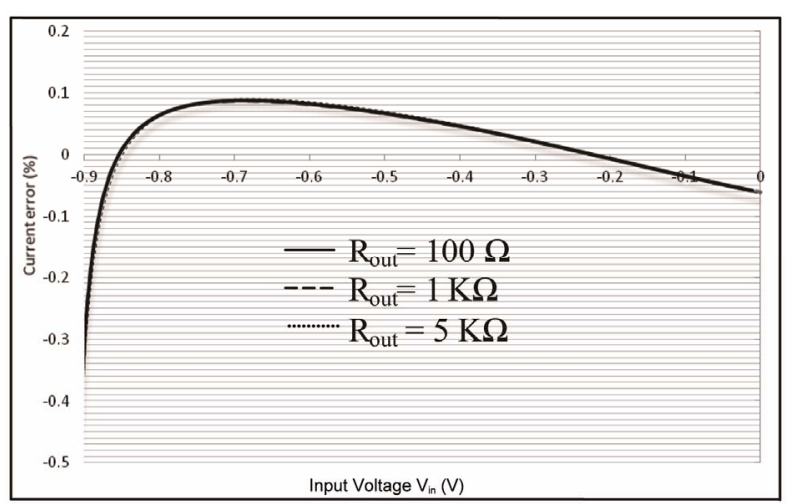

Figure 12. Current error of V-I converter.

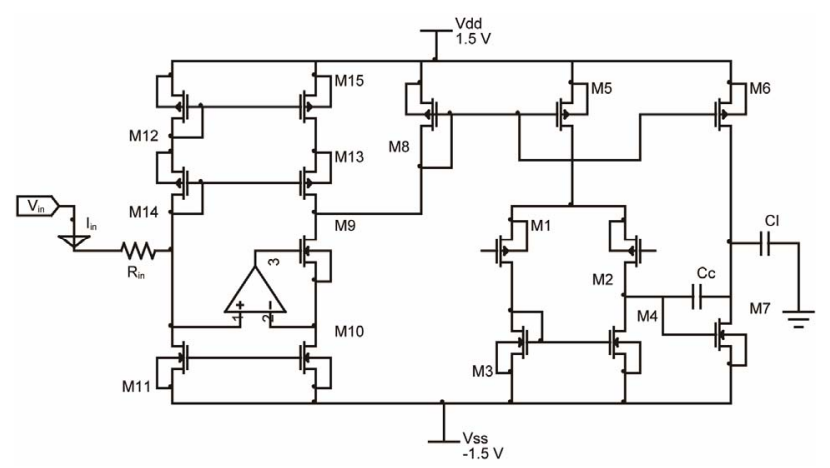

Figure 13. Proposed two-stage operational amplifier with proposed version 1 of $\mathrm{V}$-I converter. 


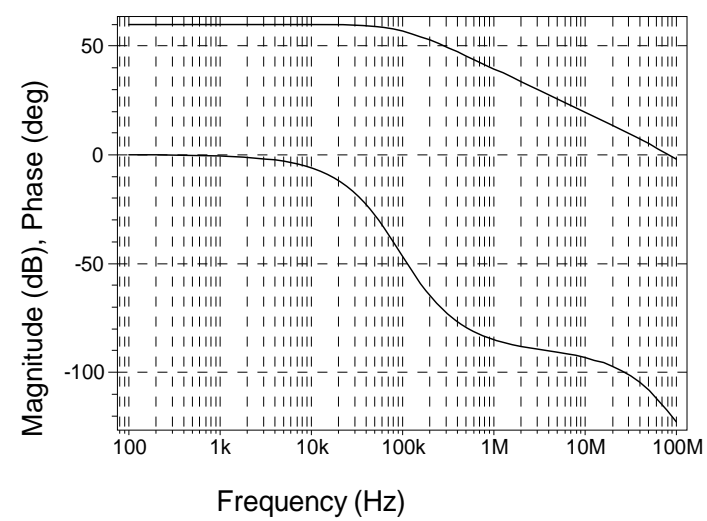

Figure 14. Frequency response of proposed V-I converter.

\section{Conclusion}

Current mirror play an important role in analog circuits used for V-I converter. This work presents two novels design of V-I converter. The version 1 of V-I converter is implemented in Two-Stage Operational Amplifier. However the version 2 gives high output impedance. The use of version 1 due to its large bandwidth of $750 \mathrm{MHz}$ and gain of $80.2 \mathrm{~dB}$. Simulations results of application of V-I converter in two-stage operational amplifier indicated that phase margin is $62^{\circ}$ to ensure a good stability, gain of $60 \mathrm{~dB}$ for $\pm 1.5 \mathrm{~V}$, and $\mathrm{GBW}$ of $82 \mathrm{MHz}$.

\section{REFERENCES}

[1] M. H. Li and H. L. Kwork, "The Application of CurrentMode Circuits in the Design of an A/D Converter," IEEE Canadian Conference on Electrical and Computer Engineering, Vol. 1, 1998, pp. 41-44.

[2] K.-H. Cheng, C.-C. Chen and C.-F. Chung, "Accurate Current Mirror with High Output Impedance," 8th IEEE International Conference Electronics on Circuits and
Systems, Vol. 2, 2001, pp. 565-568.

[3] K.-H. Cheng, T.-S. Chen and C.-W. Kuo, "High Accuracy Current Mirror with Low Settling Time," Proceedings of the 46th IEEE International Midwest Symposium on Circuits and Systems, Vol. 1, 2003, pp. 189-192.

[4] M. S. Sawant, J. Ramirez-Angulo, A. J. Lopez-Martin and R. G. Carvajal, "New Compact Implementation of a Very High Performance CMOS Current Mirror," 48th Midwest Symposium on Circuits and Systems, Vol. 1, 2005, pp. 840-842. doi:10.1109/MWSCAS.2005.1594232

[5] J. Ramirez-Angulo, R. G. Carvajal and A. Torralba, "Low Supply Voltage High Performance CMOS Current Mirror with Low Input and Output Voltage Requirements," IEEE Transactions on Circuits and Systems-II Express Briefs, Vol. 51, No. 3, 2004, pp. 124-129.

[6] A. N. Mohieldin, E. Sánchez-Sinencio and J. SilvaMartínez, "Nonlinear Effects in Pseudo Differential OTAs with CMFB," IEEE Transactions on Circuits and Systems-II: Analog and Digital Signal Processing, Vol. 50, No. 10, 2003, pp. 762-770.

[7] K. tanno, O. Ishizuka and Z. Tang, "Low Voltage and Low Frequency Current Mirror Using a Two-MOS Subthreshold op-amp," Electronics Letters, Vol. 32, No. 7, 1996, pp. 605-606.

[8] V. Srinivasan, R. Chawla and P. Haster, "Linear Current to Voltage and Voltage to Current Converters," 48th Midwest Symposium on Circuits and Systems, Vol. 1, 2005, pp. 675-678.

[9] B. H. Soni and R. N. Dhavse, "Design of Operational Transconductance Amplifier Using $0.35 \mu \mathrm{m}$ Technology," International Journal of Wisdom Based Computing, Vol. 1, No. 2, 2011, pp. 28-31.

[10] M. M. Amourach and R. L. Geiger, "Gain and Bandwidth Boosting Techniques for High-Speed Operational Amplifiers," IEEE International Symposium on Circuits and Systems, Vol. 1, 2001, pp. 232-235. 